\title{
Adverse pregnancy outcomes associated with maternal prenatal ingestion of traditional medicine
}

\author{
A Siveregi, ${ }^{1}$ MB ChB, MSc Clinical Epi, PG Dip Occ Health; N C Ngene, ${ }^{1,2}$ MBBS, Dip Obst (SA), Dip HIV Man (SA), MMed \\ (Fam Med), FCOG (SA), MMed (Obstetr Gynaecol), PhD \\ ${ }^{1}$ Department of Obstetrics and Gynaecology, Klerksdorp-Tshepong Hospital Complex, Klerksdorp, South Africa \\ ${ }^{2}$ Department of Obstetrics and Gynaecology, School of Clinical Medicine, Faculty of Health Sciences, University of the Witwatersrand, \\ Johannesburg, South Africa
}

Corresponding author: N C Ngene (ngenenc@gmail.com)

\begin{abstract}
Pregnancy is associated with complications ranging from minor ailments to major morbidity and mortality. To prevent such complications, some women, including some in South Africa, resort to the use of traditional medicines. These are meant to either supplement or replace conventional medicines that are offered by their healthcare facilities. Some of these medicines, however, have the potential to cause harm, can increase pregnancy-related complications and may adversely interact with other medicines prescribed during the pregnancy. We present a case of an 18-year-old primigravida who commenced prenatal ingestion of traditional medicine (moruto wamfene, otherwise called baboon urine) at 28 weeks' gestation, in an attempt to improve her pregnancy outcomes. However, she instead developed uterine hyperstimulation, fetal bradycardia and thick meconium-stained liquor during labour. This report is intended to raise awareness about prenatal ingestion of traditional medicine (particularly moruto wamfene), highlights the safety concerns and suggests preventive measures.
\end{abstract}

S Afr J Obs Gynae 2019;25(1):6-8. DOI:10.7196/SAJOG.2019.v25i1.1423

The use of traditional medicine in pregnancy is practised globally, particularly in many low- and middle-income countries, possibly as a result of inadequate healthcare coverage. The World Health Organization defines traditional medicine as the sum total of the knowledge, skills and practices based on theories, beliefs and experiences indigenous to different cultures, whether explicable or not, used in the maintenance of health as well as in prevention, diagnosis, improvement or treatment of physical and mental illnesses. ${ }^{[1]}$ In South Africa (SA), despite the fact that pregnant women have access to conventional medicine, some still use traditional medicine, for reasons that may include indications that they feel are inadequately or not at all covered by conventional healthcare. These indications include protection from evil spirits during pregnancy, preparation of the pelvis and uterus for labour and prevention of colic and other ailments in the baby. ${ }^{[2]}$ Herbal preparations are used at various stages of pregnancy, from conception to delivery, to achieve a specific favourable pregnancy outcome. ${ }^{[2]}$

Isihlambezo is a broad term that refers to various liquid herbal mixtures taken by some women in the KwaZulu-Natal Province of SA to help them to conceive, prepare the uterus for pregnancy, maintain uterine quiescence during pregnancy, promote cervical ripening and uterine contractions at term and prevent postpartum haemorrhage, among many other uses. ${ }^{[2]}$ Of concern, however, is that of the approximately 60 plant species used in isihlambezo mixtures, some have been implicated in human fatalities. For instance, Rhoicissus tridentate extracts have been linked to fatalities, through their ability to cause central nervous system depression and respiratory failure. ${ }^{[3]}$
In different parts of SA, these types of traditional medicines bear other names and may vary in method of preparation, but there remains a paucity of data about their constituents, safety and efficacy. There is also concern that they may cause adverse pregnancy outcomes. We present a case of a woman in SA who frequently ingested a traditional medicine, moruto wamfene (a Tswana term meaning 'baboon urine'), during the antenatal period to improve the outcome of her pregnancy, but who subsequently developed uterine hyperstimulation, meconium-stained liquor and fetal compromise during labour. This report raises concerns about the safety of prenatal ingestion of unlicensed traditional medicine.

\section{Case report}

An 18-year-old primigravida residing with her parents and siblings commenced antenatal care in a primary healthcare clinic in SA at 10 weeks' gestation. During the first antenatal care clinic visit, she had no symptoms. She had consumed alcohol previously, but not in pregnancy. The physical examination and routine antenatal laboratory investigations were normal, and she was placed on prenatal vitamins. She had a total of 6 antenatal care clinic visits, and at 28 weeks' gestation started ingesting moruto wamfene, a traditional medicine bought from a herbal pharmacy, which according to the patient was meant to protect her from evil spirits during pregnancy, prepare her for labour, promote fetal health and prevent colic in the baby after delivery. She said that the medicine was bought as chunks of dark brown solid material. Small pieces of the medicine were broken off and added to a litre of cold water, which she drank once a week. According to the patient, the solution smells like animal excreta, but has no side-effects. She had been 
introduced to the medicine by her mother, who had used it in all her pregnancies with no reported complications. The patient claimed to know many other antenatal clinic attendees who took the same medicine.

At 39 weeks' gestation, the patient's routine repeat HIV test was positive (CD4 count 1690 cells $/ \mathrm{mm}^{3}$ ), and she commenced a fixed-dose combination antiretroviral drug containing tenofovir, emtricitabine and efavirenz. The next day, she presented at the primary healthcare clinic in the latent phase of labour, having taken the traditional medicine. The fetus was in cephalic presentation, and cervix $2 \mathrm{~cm}$ dilated, with the membranes intact. Routine intrapartum care was commenced. Subsequently, the patient had spontaneous rupture of the membranes that revealed thick meconium (grade 2), and she was transferred to hospital for further care.

On arrival at the hospital, she had 6 uterine contractions in 10 minutes; the cardiotocograph showed fetal bradycardia, and the cervix was $5 \mathrm{~cm}$ dilated. A diagnosis of uterine hyperstimulation and fetal compromise was made, and intrapartum resuscitation, including tocolysis, was commenced. She was subsequently booked for an emergency caesarean delivery. There were no delays associated with her management. She had an uneventful caesarean section and was delivered of a normal baby boy, with a 5-minutes Apgar score of 8 and birth weight of $3.085 \mathrm{~kg}$. The placenta appeared normal, except for the meconium stains. The placental histology and umbilical blood gas were erroneously not requested by the attending medical officer. The baby was commenced on prophylactic antiretroviral therapy. The patient's clinical condition (including laboratory investigations - full blood count, blood urea and creatinine and liver function tests) remained normal, and she was discharged home with her baby 3 days after delivery. Postpartum, the followup reviews showed that the baby and her mother remained normal. Counselling on contraception and health help-seeking behaviour was provided.

Written informed consent was obtained from the patient for publication of this case report.

\section{Discussion}

Uterine hyperstimulation is defined by contractions that are either $>5$ in number within 10 minutes, or that exceed 2 minutes in duration. ${ }^{[4]}$ It is a risk factor for fetal compromise and postpartum haemorrhage. The latter is one of the leading causes of maternal mortality in SA. In the index case, the uterine hyperstimulation and fetal compromise were associated with ingestion of moruto wamfene, and these complications are known adverse outcomes of traditional medicine use in pregnancy. ${ }^{[2]}$ Placental histology and umbilical blood gas would have provided additional information. Nonetheless, other reported outcomes of traditional medicine use in pregnancy are preterm labour, oligohydramnios, unexplained fetal death, low birth weight and neonatal jaundice. ${ }^{[3]}$ These deleterious effects are unsurprising, given that the dosages of many of these traditional medicines are largely arbitrary. Anecdotal evidence seen and heard by the authors showed that moruto wamfene, just like isihlambezo, comes in different forms, but the dark-brown formulation that needs dissolution in water before ingestion is common (Fig. 1). The patient in the present case was given instructions to break off a small piece from a chunk and dissolve it in clean water before ingestion. The size of a 'small piece' will depend on the user, and the amount of active chemical constituents in the chosen dose may be variable, and largely unknown. This arbitrariness alone puts the lives of patients at risk.

The interaction of traditional medicines with other therapies such as antiretroviral drugs remains unknown, and is of concern. This is an important consideration, given that the patient was on antiretroviral therapy. Part of the community health education provided to pregnant women should, therefore, include advice against the use of unlicensed medications whose constituents and effects are not formally known. This is of particular importance in
SA, where $30.8 \%$ of pregnant women are HIV-infected, ${ }^{[5]}$ and many of them are on antiretroviral therapy.

A study conducted in a tertiary health facility in SA over 20 years ago estimated the prevalence of traditional medicine use in pregnancy to be approximately $55 \%$ in that setting. ${ }^{[6]}$ New studies on the prevalence of usage of these medicines (particularly moruto wamfene) in pregnancy are needed. These studies should also aim to identify the chemical constituents of the traditional medicines sold under the name moruto wamfene, as there is a scarcity of data on their composition. Such studies could unravel the possible harm that may be caused by moruto wamfene. The patient declared that she knew of other women attending the antenatal clinic with her who were using similar traditional medicine, which suggests that many pregnant women may be at risk if these medicines cause harm. Additionally, it is of the utmost importance to identify and refine those traditional products with proven efficacy, and integrate them into the conventional medical industry.

\section{Conclusion}

The ingestion of the traditional medicine moruto wamfene during pregnancy was associated with adverse pregnancy outcomes such as uterine hyperstimulation and fetal compromise. Unfortunately, some medicines marketed in herbal pharmacies in SA have not been fully studied; their safety and efficacy are yet to be established, and some are associated with harm. This needs to be communicated to patients when they attend antenatal care clinics, to avoid adverse outcomes from the use of these agents. Additionally, evaluation of pregnant women in the antenatal period in SA should routinely include a history of traditional medicine use. It is also important to study

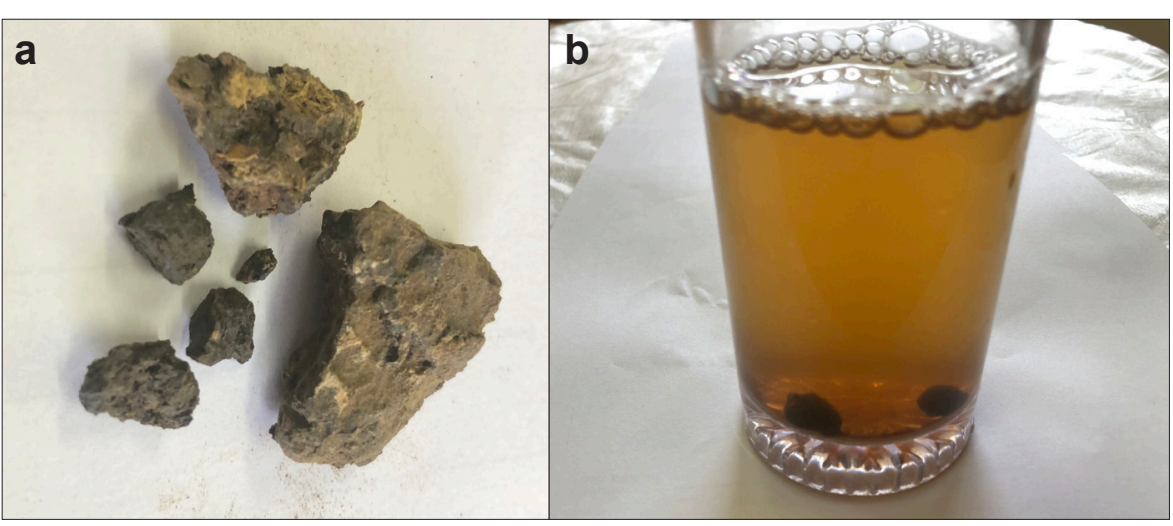

Fig. 1 a and b. Moruto wamfene (a) undissolved, and (b) dissolved in drinking water. 


\section{CASE REPORT}

these medicines and incorporate those that are safe and effective into the formal healthcare system.

Acknowledgements. The authors would like to thank the patient, who consented to have the case written up and published.

Author contributions. NCN conceived the idea to report the case. AS and NCN made equal contributions to writing and revising the manuscript.

Funding. None.

Conflicts of interest. None.

1. World Health Organization. Traditional, complementary and integrative medicine. http://www. who.int/traditional-complementary-integrative-medicine/about/en/ (accessed 11 November 2018).
2. Varga CA, Veale DJ. Isihlambezo: Utilisation patterns and potential health effects of pregnancyrelated traditional herbal medicine. Soc Sci Med 1997;44(7):911-24. https://doi.org/10.1016 s0277-9536(96)00104-9

3. Brookes KB, Smith AN. Cytotoxicity of pregnancy-related traditional medicines. S Afr Med J 2003;93(5):359-361

4. National Institute for Health and Clinical Excellence. Induction of labour (Clinical Guidelines No. 70) 2008. https://www.nice.org.uk/guidance/cg70/ evidence/cg70-induction-of-labour-fullguideline2 (accessed 11 November 2018).

5. National Department of Health, South Africa. The 2015 National Antenatal Sentinel HIV and Syphilis Survey Report 2017. http://www.health.gov.za/index.php/ shortcodes/2015-03-29-10Syphilis Survey Report 2017. http://www.health.gov.za/index.php/ shortcodes/2015-03-29-10$42-47 / 2015-04-30-08-18-10 / 2015-04-30-08-21$ - 56 ? download=
prevalence-survey-final-23oct17 (accessed 10 November 2018)

6. Mabina MH, Moodley J, Pitsoe SB. The use of traditional herbal medication during pregnancy. Trop Doct 1997;27(2):84-86. https://doi.org/10.1177/004947559702700212

Accepted 27 July 2019. 